\title{
Clinical Application of Computed Radiography in Orthopedic Surgery
}

\author{
Satoru Fujita, Masamichi Tanaka, Sigeaki Hirota, and Takeshi Fuji
}

\begin{abstract}
Since 1988, Fuji Computed Radiography (FCR) system (Fuji Medical Systems, Tokyo, Japan) has been used at Osaka Prefectural Hospital (Osaka, Japan) for all kinds of images. In this paper, we discuss the advantages and disadvantages of computed radiography (CR) images from the standpoint of an orthopedic surgeon. Contours, which can not be seen on conventional radiographs, are clearly visualized on the CR image. Adequate information for diagnosis can be obtained with a great reduction in $x$-ray exposure during the screening of scoliosis or congenital dislocation of hip joint. However, because the scale is reduced by one half in antero-posterior views of the bilateral hip joint, CR images are unsuitable for postoperative measurement of total hip arthroplasty (THA). Furthermore, caution is required because the clear zone after THA is emphasized on edge-enhanced CR image.

Copyright 1995 by W.B. Saunders Company
\end{abstract}

KEY WORDS: computed radiography, orthopedic surgery.

$\mathbf{I}^{\mathbf{N}}$ N SEPTEMBER 1988, the Fuji Computed Radiography (FCR) system ${ }^{1}$ (Fuji Medical Systems, Tokyo, Japan) was introduced at Osaka Prefectural Hospital (Osaka, Japan) for all kinds of images. This was the first time such a system had been used anywhere in the world and it attracted great attention. In this report, we will discuss the advantages and disadvantages of computed radiography (CR) images from the standpoint of an orthopedic surgeon. Because we do not intend to compare CR images with conventional screen-film images, we will limit the discussion to our impressions concerning CR images. We will first discuss CR images of the spine and hip joints, and then introduce CR dual-energy x-ray absorptiometry $^{2}$ (CR-DXA), a new method of measuring bone mineral density using FCR.

\section{CERVICAL SPINE}

One hard copy with two CR images that is routinely supplied in our hospital is shown in Fig 1 . The left image was processed to simulate conventional radiography with gradation processing along the S-curve (Fig 2A). We call this image "default image" in this report. The right image was processed with significant edge enhancement and linear gradation processing (Fig 2B). On this image, the margins of the bone and soft tissue were stressed, and will be referred to as "edge-enhanced image" in this report.

Case 1 with a 66 body fracture is shown in Fig $3 \mathrm{~A}$. Cervical instability caused by burst fracture of $\mathrm{C} 6$ was clearly seen on dynamic lateral views because the contours of the spinous process are clear in the edge-enhanced image. Because no compression of the spinal cord was seen in myelography and computed tomography myelography, posterior spinal fusion with bone grafting using an Alligator plate was performed (Fig 3B). The grafted bone union, seen on an oblique view, can also be easily confirmed by the edge-enhanced image (Fig 3C).

\section{THORACIC SPINE}

Many patients with the multiple metastases in the thoracic spine come to see us and complain of back pain and/or paraparesis. In case 2, the right pedicle of the Th 12 vertebra had disappeared and a so-called pedicular wink was evident in the edge-enhanced image (Fig 4). This case proved to have Gravitz tumor.

At our hospital, CR is obtained with approximately one fifth of the radiation dose for conventional radiography in cases of scoliosis. In case 3 , the CR image showed poor granularity because of the reduced dose (Fig 5A). However, the contours of the vertebral bodies were clear enough to measure the Cobb angle (Fig 5B). Thus, the $\mathrm{x}$-ray dose can be reduced in such young patients.

From the Department of Orthopedic Surgery, Osaka Prefectural Hospital, Osaka, Japan.

Address reprint requests to Satoru Fujita, MD, Department of Orthopedic Surgery, Osaka Prefectural Hospital, 3-1-56, Mandai-Higashi, Sumiyoshi-ku, Osaka 558, Japan.

Copyright $(1995$ by W.B. Saunders Company

0897-1889/95/0801-1013\$3.00/0 


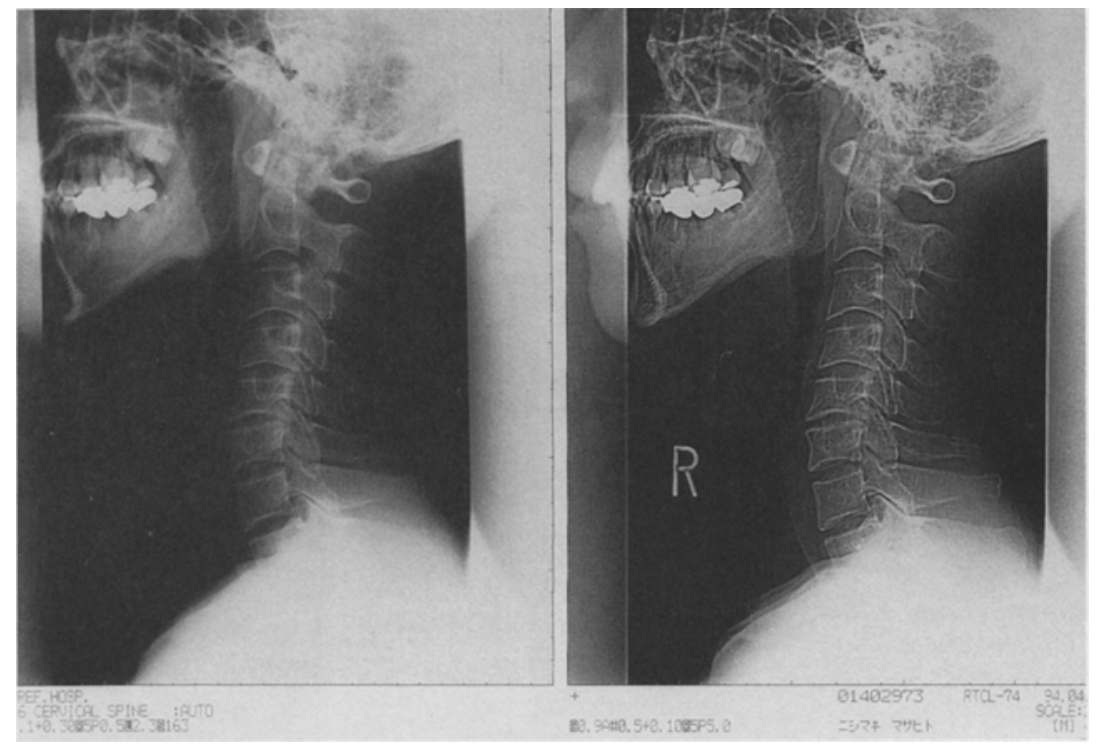

Fig 1. An x-ray film of the cervical spine processed at Osaka Prefectural Hospital.

\section{LUMBAR SPINE}

In the lateral radiograph of the lumbar spine, the contour of L5 vertebral body is often unclear because of overlapping iliac bones. In the default image of case 4 , the L5 body was not clear (Fig 6A), but in the edge-enhanced image of the same case, the L 5 body could be seen and the spinous process was also clear (Fig 6B).

In October 1988, the FCR system was also introduced into the operating room. The sur-

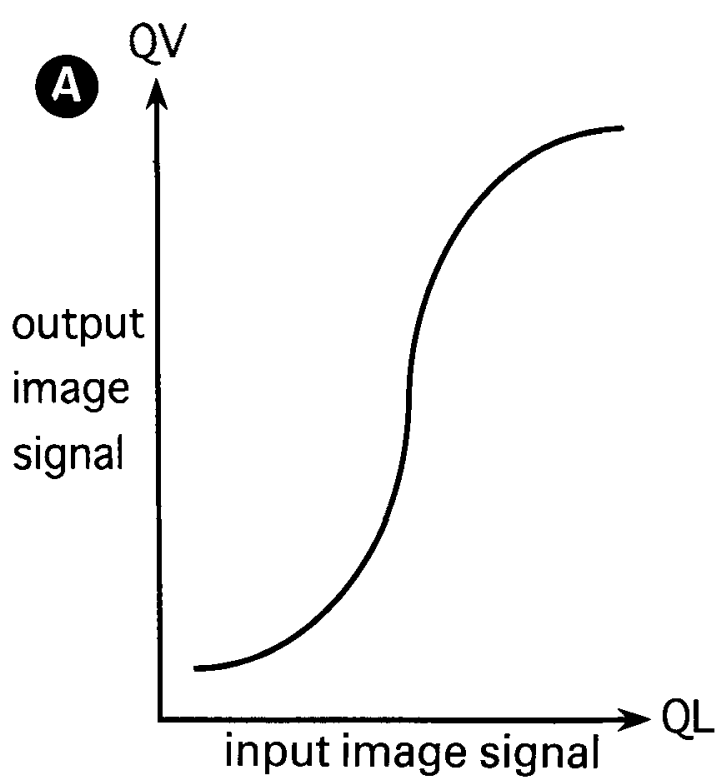

geon can see the intraoperative control radiograph on the monitor immediately after exposure. Because gradation processing can be adjusted on the monitor, there was no error in diagnosing the level of the spinous process even in obese patients. On the conventional film in case 5 , the contour of the spine cannot be seen because of obesity (Fig 7A). But on the edgeenhanced image, we can see the result of surgery despite using the same portable $x$-ray

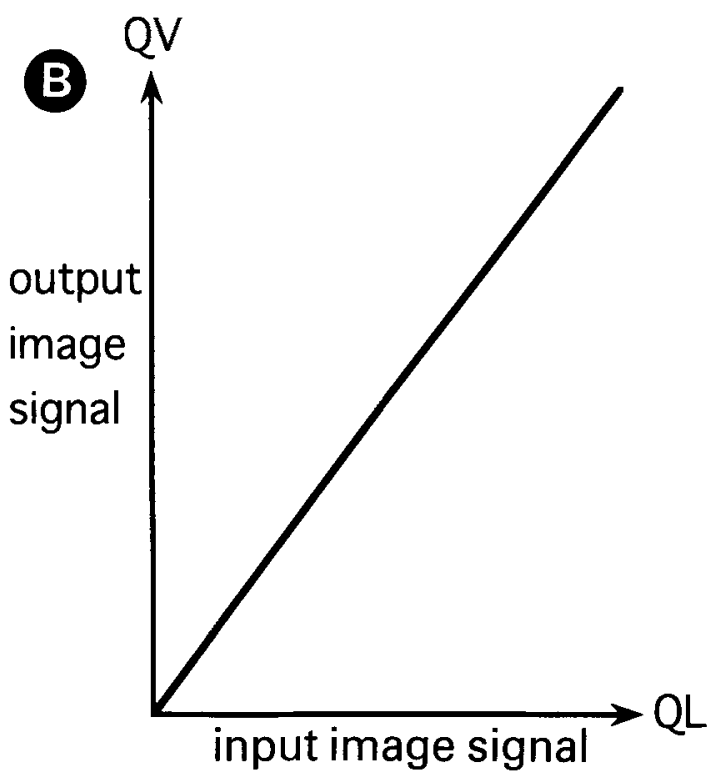

Fig 2. Gradation processing along the S-curve (A) and linear gradation (B). 

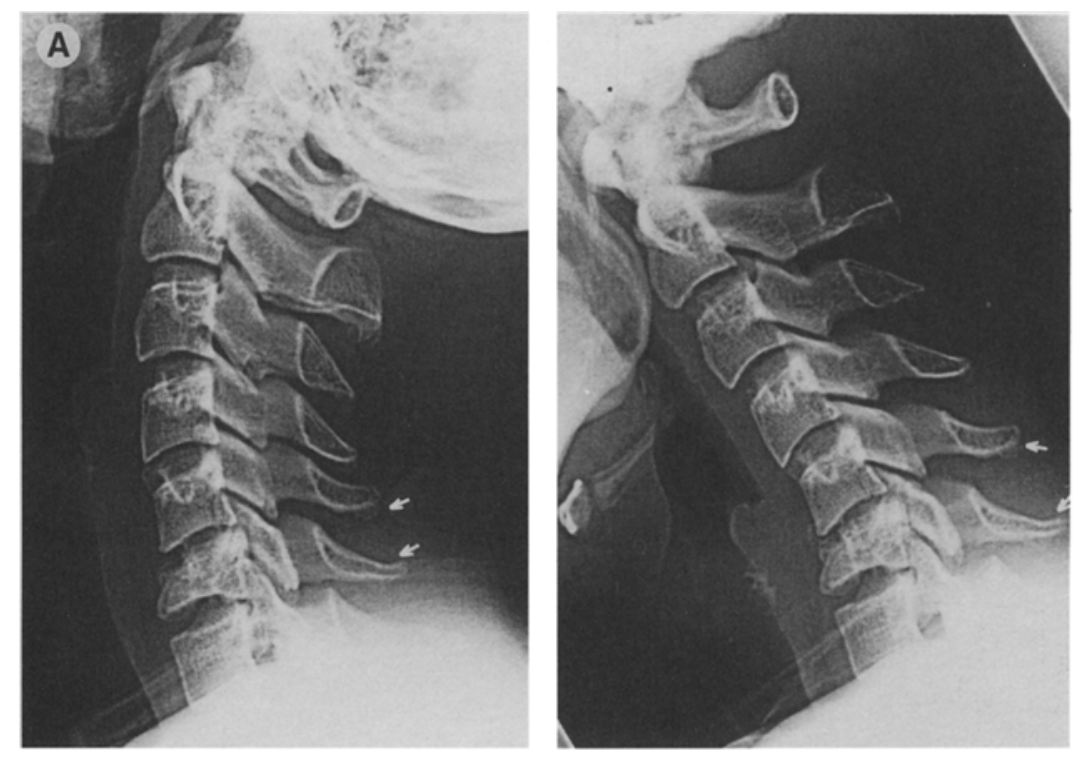

Fig 3. Case 1: Lateral view of a C6 body fracture (A). Posterior spinal fusion with bone grafting using Alligator plate was per-
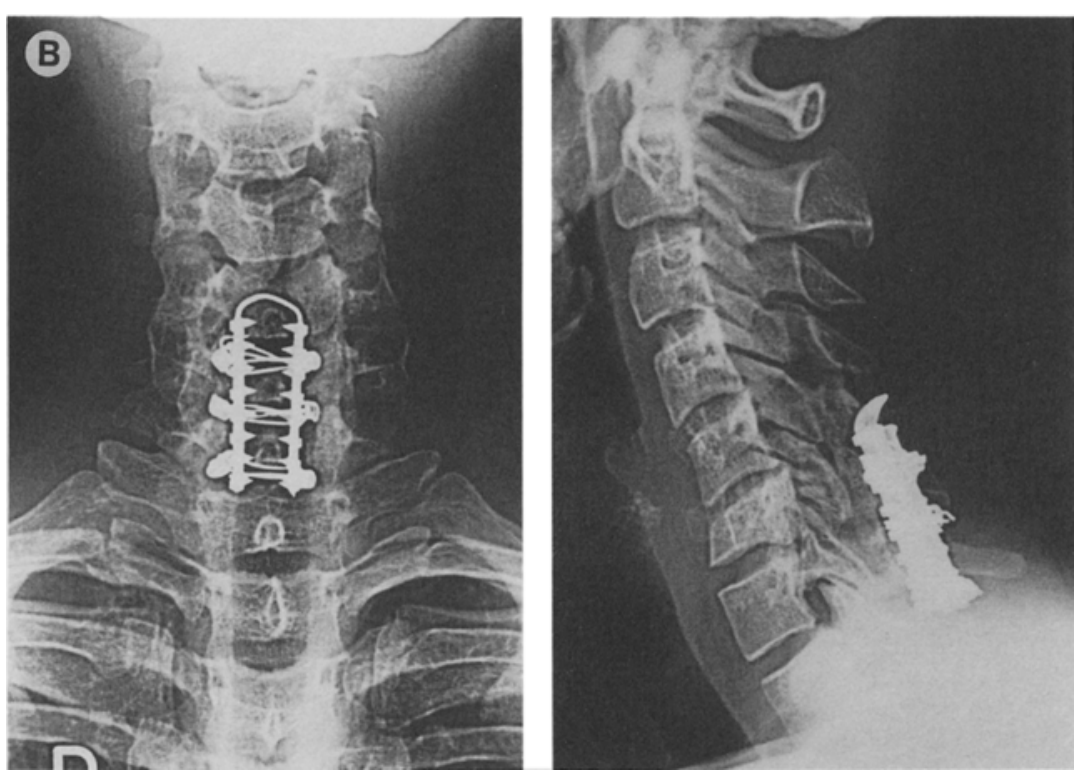
formed (8).

irradiation system as used for the previous image (Fig 7B).

\section{HIP JOINT}

First, in pediatric cases, the hip is imaged by the varying $x$-ray dose depending on the disease suspected. In screening for congenital dislocation or acetabular dysplasia of hip joint, sufficient image quality for diagnosing these conditions can be obtained with edge-enhanced images acquired with one fifth of the radiation dose. The image in case 6 showed an evident congenital dislocation of the right hip joint from the positional relation of the pelvis and femur (Fig 8). However, when Perthes disease is suspected as in case 7 (Fig 9), a clear image of the epiphysis of the femoral head is required. Therefore, radiation dose as same as the dose for conventional screen-film radiography is recommended.

In adult case, the next discussion concerns postoperative evaluations of total hip arthroplasty (THA). The most important point in postoperative evaluation is to check for loosen- 

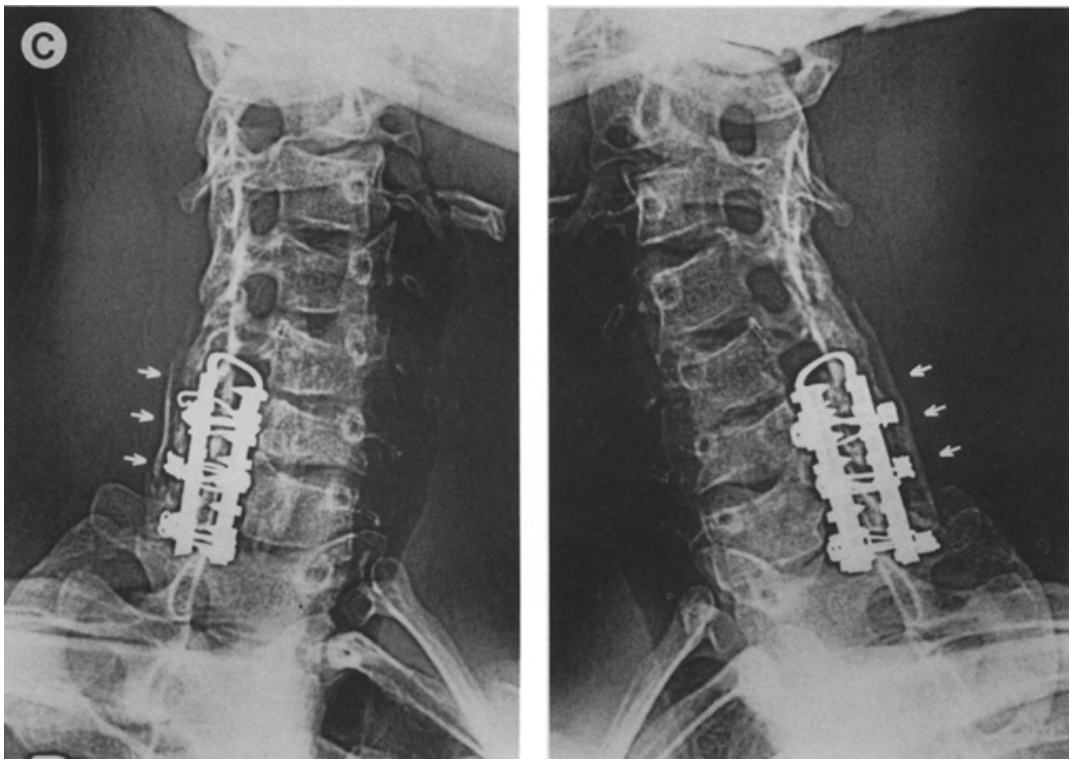

Fig 3. (Cont'd). Union of the grafted bone seen on the oblique view was easily confirmed (C). ing of the implant. Loosening of the implant is diagnosed from migration of the implant and/or a clear zone around the implant. If the center of the head has immigrated more than $3 \mathrm{~mm}$ vertically or horizontally, migration of the cup is definite. The antero-posterior view of the bilateral hip joint is necessary to measure migration (Fig 10). However, on an edge-enhanced image, the chance of the measurement errors increases because the scale is reduced by one half. Case 8 showed upper migration of the cup 8 years after surgery (Fig 11), and loosening was diagnosed.

When a clear zone more than $2 \mathrm{~mm}$ is observed around the implant, loosening is diagnosed. On edge-enhanced images, overreading tends to occur because the clear zone is emphasized. In case 9 , there was a definite clear zone around the cup on the edge-enhanced image (Fig 12A), but on the default image, it was evaluated as a radiolucent line with no relation
Fig 4. Case 2: The right pedicle of the Th 12 vertebra had disappeared and a so-called pedicular wink was evident farrow).

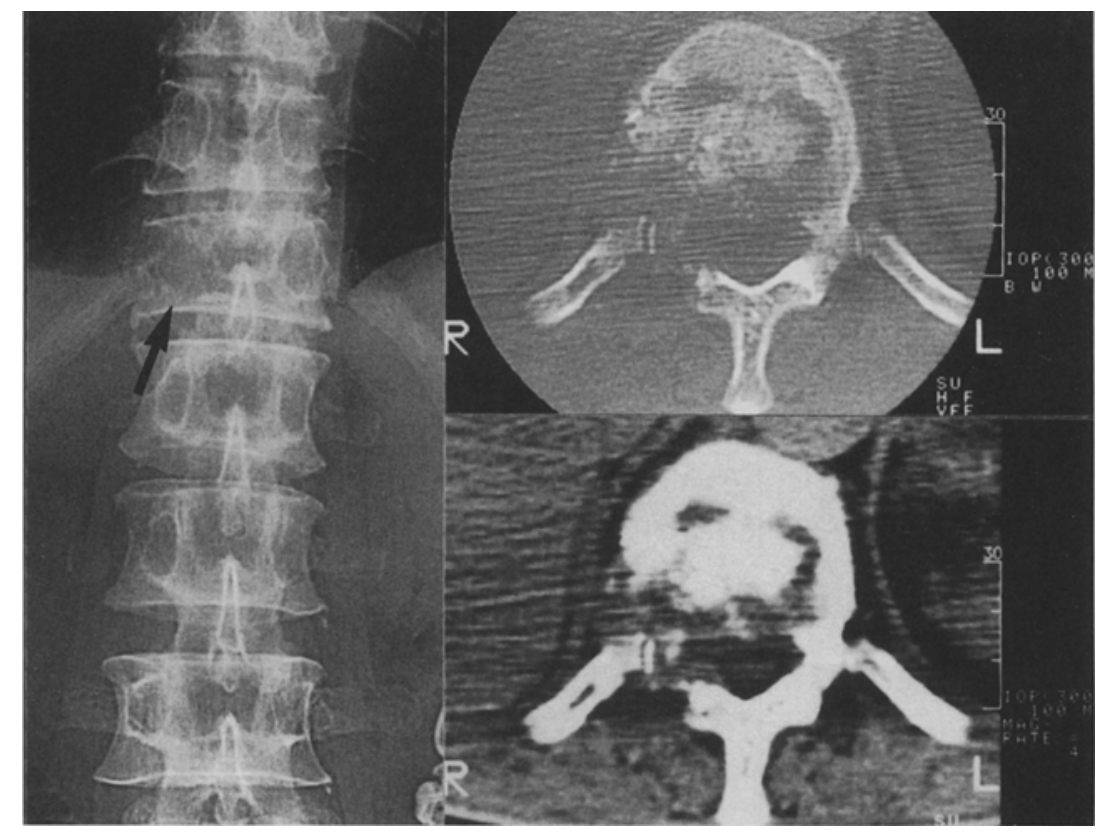




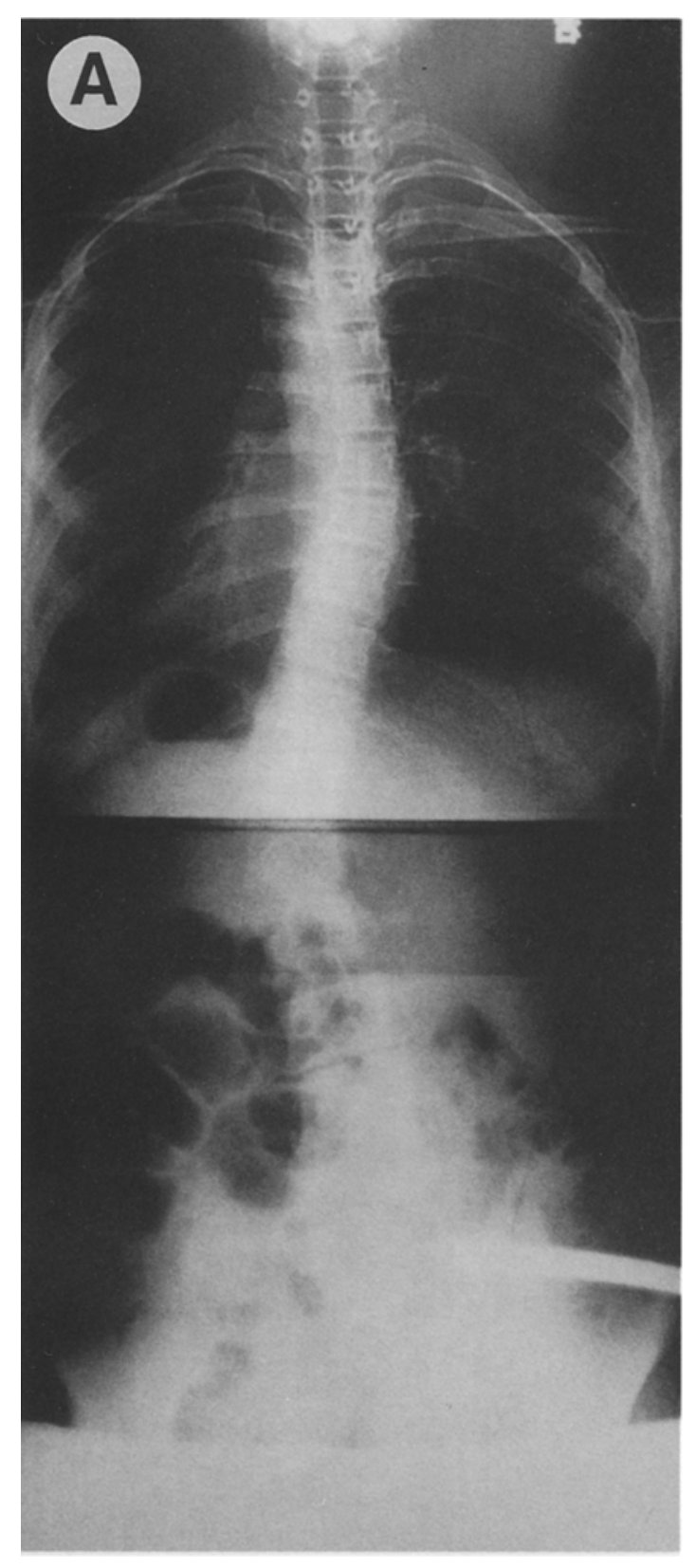

to loosening (Fig 12B). In case 10, there was no actual clear zone on default image (Fig 13A), but a radiolucent line was noted around the stem on edge-enhanced image (Fig 13B). This case proved to have no loosening. For these reasons, the clear zone should be evaluated on default images.

\section{CR-DXA}

Recently DXA has become the most accurate and widely used method of bone mineral assay,

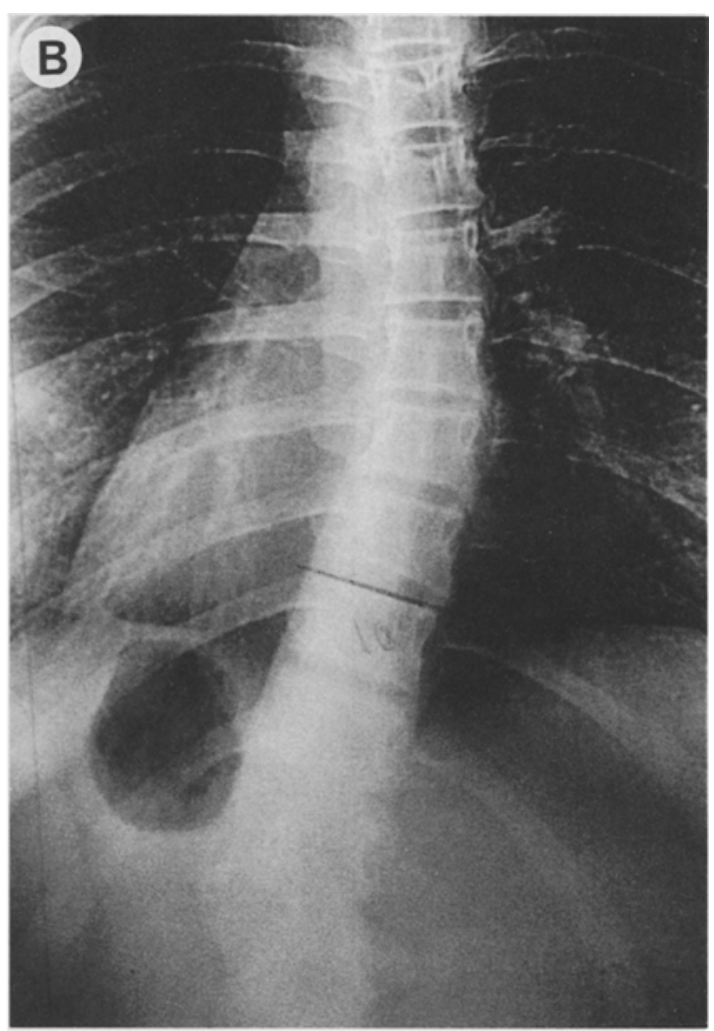

Fig 5. Case 3. Radiography was obtained with about one fifth of the normal $x$-ray dose in a case of scoliosis (A) and magnified image is also shown (B).

but it has a disadvantage in that measurement requires 5 to 8 minutes because of the pencilbeam scan of X-ray. CR-DXA is a new bone densitometry method using FCR and it features a short measurement time.

In CR-DXA (Fig 14), The calcaneus, the object to be imaged, and a bone mineral reference having a known bone mineral density (BMD) are imaged using dual-energy x-rays. The two types of CR images obtained are subjected to a weighted subtraction processing, 

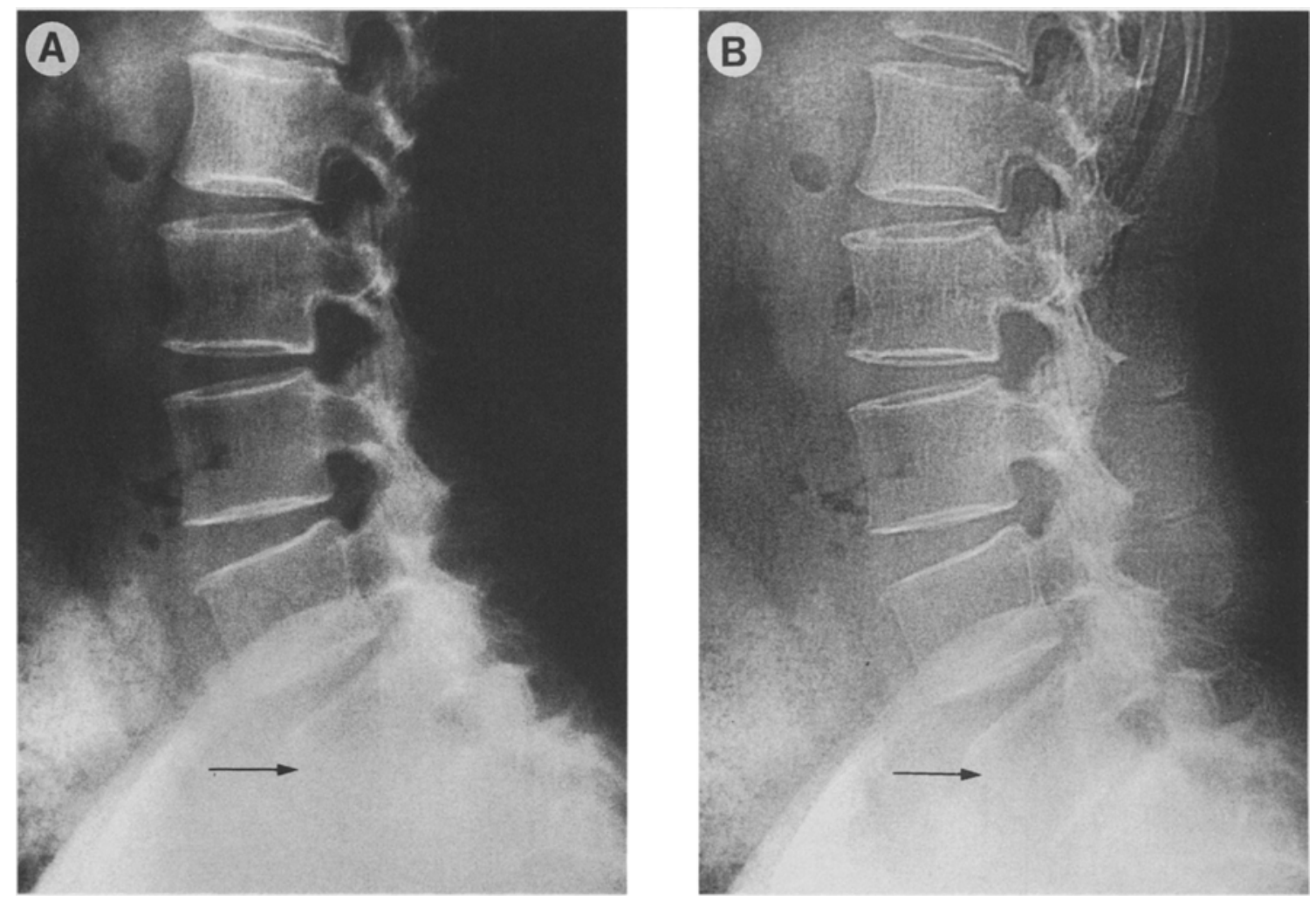

Fig 6. Case 4: Lateral view of the L5 body (arrow) on the default image (A) and on the edge-enhanced image (B)
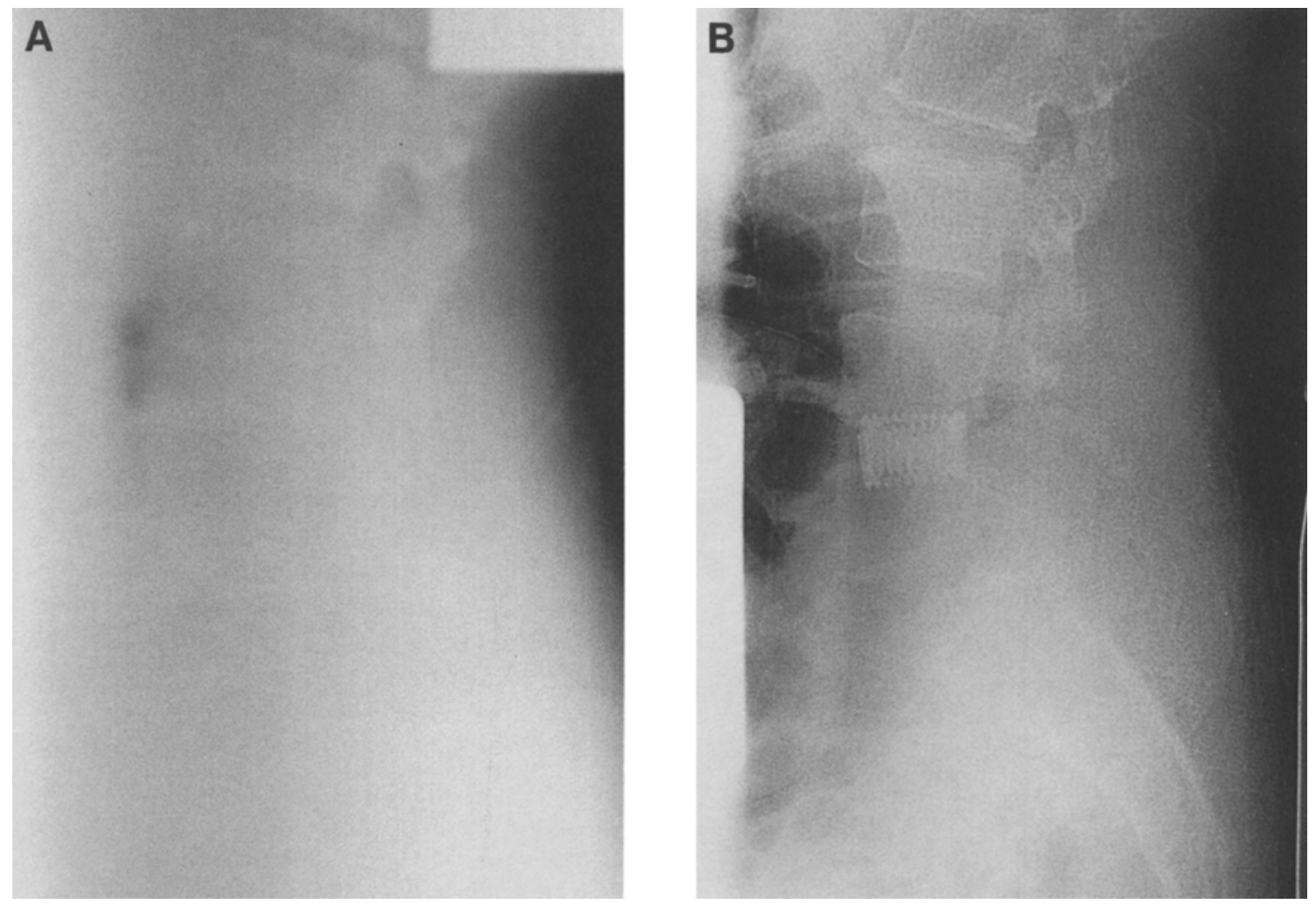

Fig 7. Case 5: Conventional screen-film radiograph in an obese patient $(27 \mathrm{mAs}$, $95 \mathrm{kYp}$; This exposure dose was the maximum obtainable with the portable $x$-ray irradiation system) (A) and an edge-enhanced image with the same exposure dose (B). 


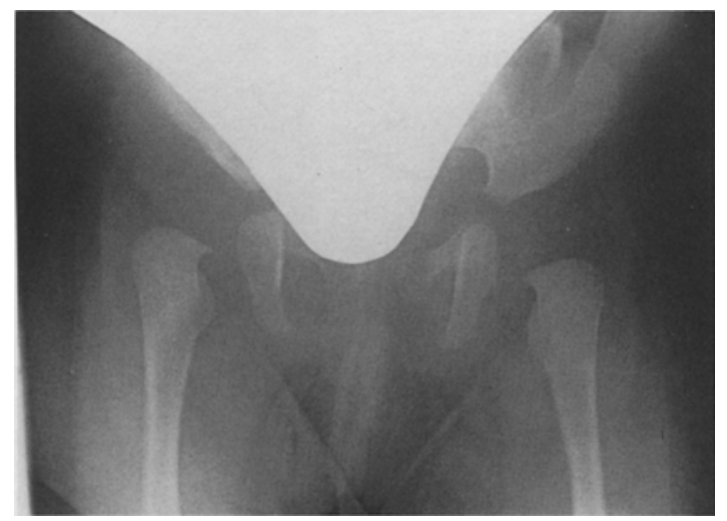

Fig 8. Case 6: Congenital dislocation of the right hip joint.

and a soft-tissue subtracted image is prepared. Because the signal-to-BMD calibration function is determined from the signal level of bone mineral reference in the soft-tissue subtracted image, the BMD is calculated based on the mean signal level in the region of interest (ROI).

The total $\mathrm{x}$-ray exposure dose is $35 \mu \mathrm{Sv}$. This is about one half of the usual $\mathrm{x}$-ray dose for a lateral view of the calcaneus. The time required for the imaging is approximately 5 seconds. We established an ROI in the calcaneus (Fig 15), and made comparative studies between conventional DXA and CR-DXA. The results of measuring BMD 5 times by each method using the calcaneus of a cadaver indicated coefficients of variation (CV) of $0.57 \%$ for DXA and $0.94 \%$ for CR-DXA. There was high repetitive preci-

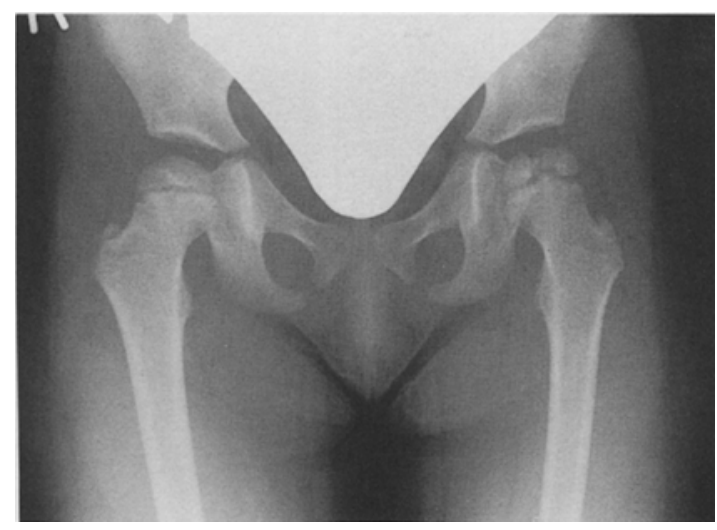

Fig 9. Case 7: Perthes disease of the left hip joint.

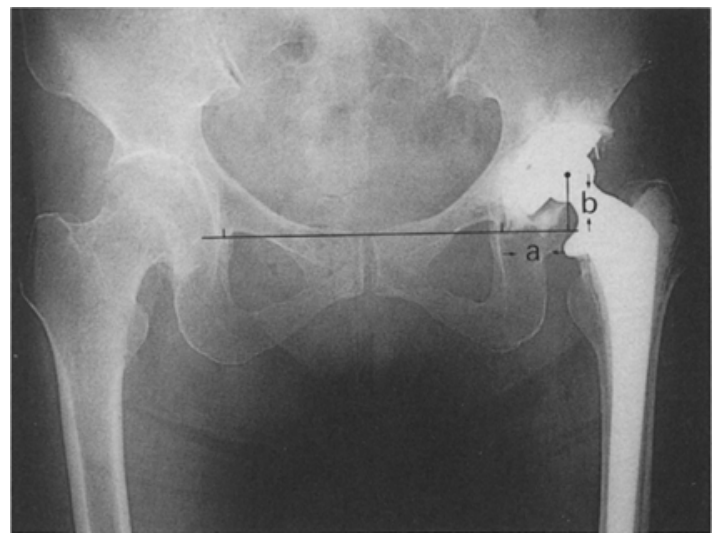

Fig 10. The method of measuring cup migration.

sion with a CV of under $1 \%$ (Table 1). Correlation of the two methods was examined in the calcaneus of 31 volunteers. The correlation was high with a regression line of $\mathrm{Y}=1.048 \mathrm{X}-$ 0.081 and a coefficient of correlation of $r=0.96$ (Fig 16). CR-DXA is a simple and accurate method for diagnosing osteoporosis.

\section{CONCLUSION}

\section{Advantages of CR Images}

Contours, which can not be seen on conventional radiographs, such as spinous processes, L5 vertebral body, and lumbar spine in obese patients, are clearly visualized on the $\mathrm{CR}$ image. Adequate information for diagnosis can be obtained with a great reduction in x-ray exposure during the screening of scoliosis or congenital dislocation of hip joint.

\section{Disadvantages of CR Images}

Because the scale is reduced by one half in $A P$ views of the bilateral hip joint, $C R$ images are unsuitable for postoperative measurement of THA. Caution is required because the clear zone after THA is emphasized during highfrequency enhancement on an edge-enhanced image.

CR-DXA, a new bone densitometry method using FCR, is a simple and accurate method for diagnosing osteoporosis. 

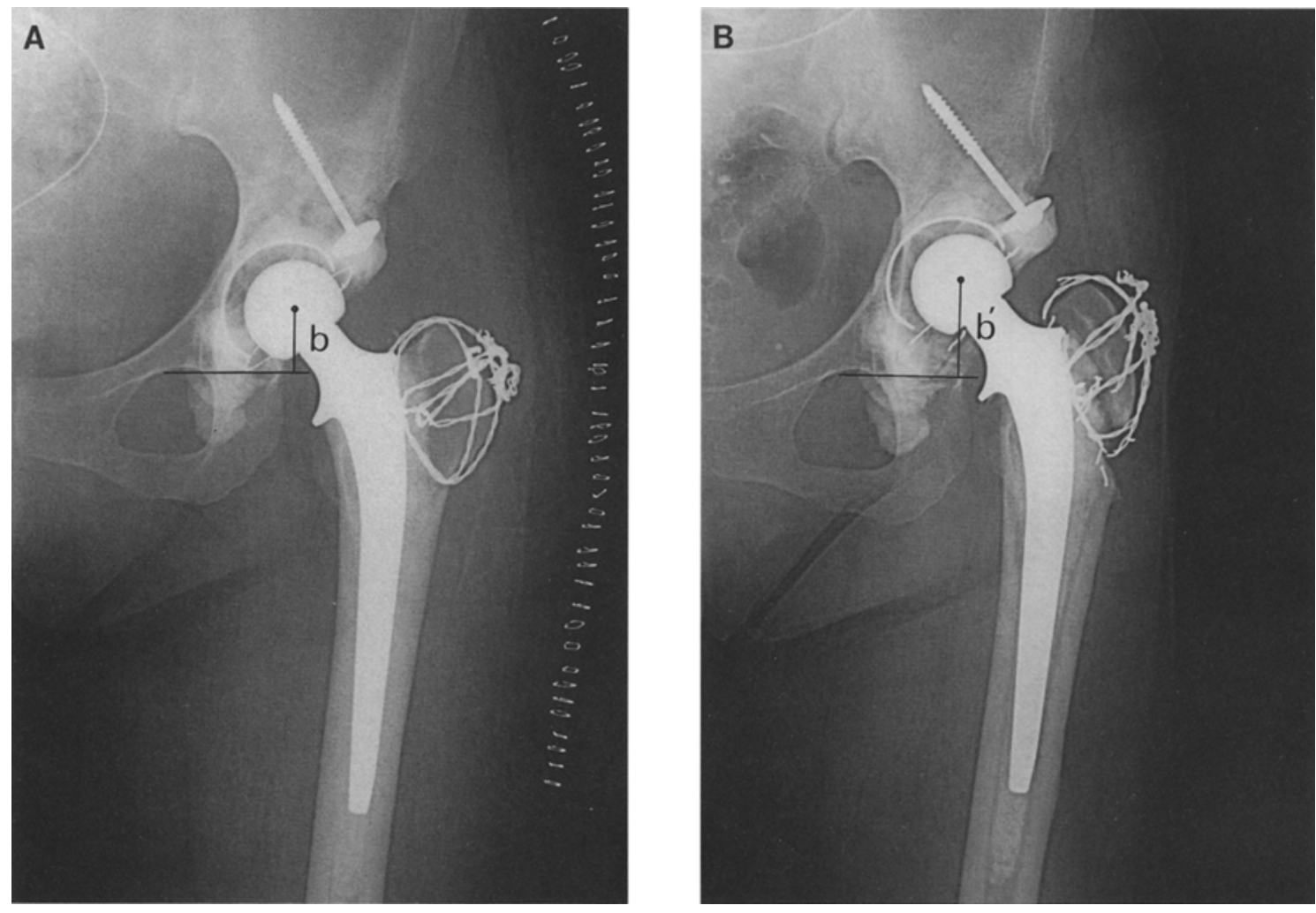

Fig 11. Case 8: Upper migration of the cup ( $\left.b<b^{\prime}\right)$ immediately after surgery $(A)$ and 8 years after surgery (B).
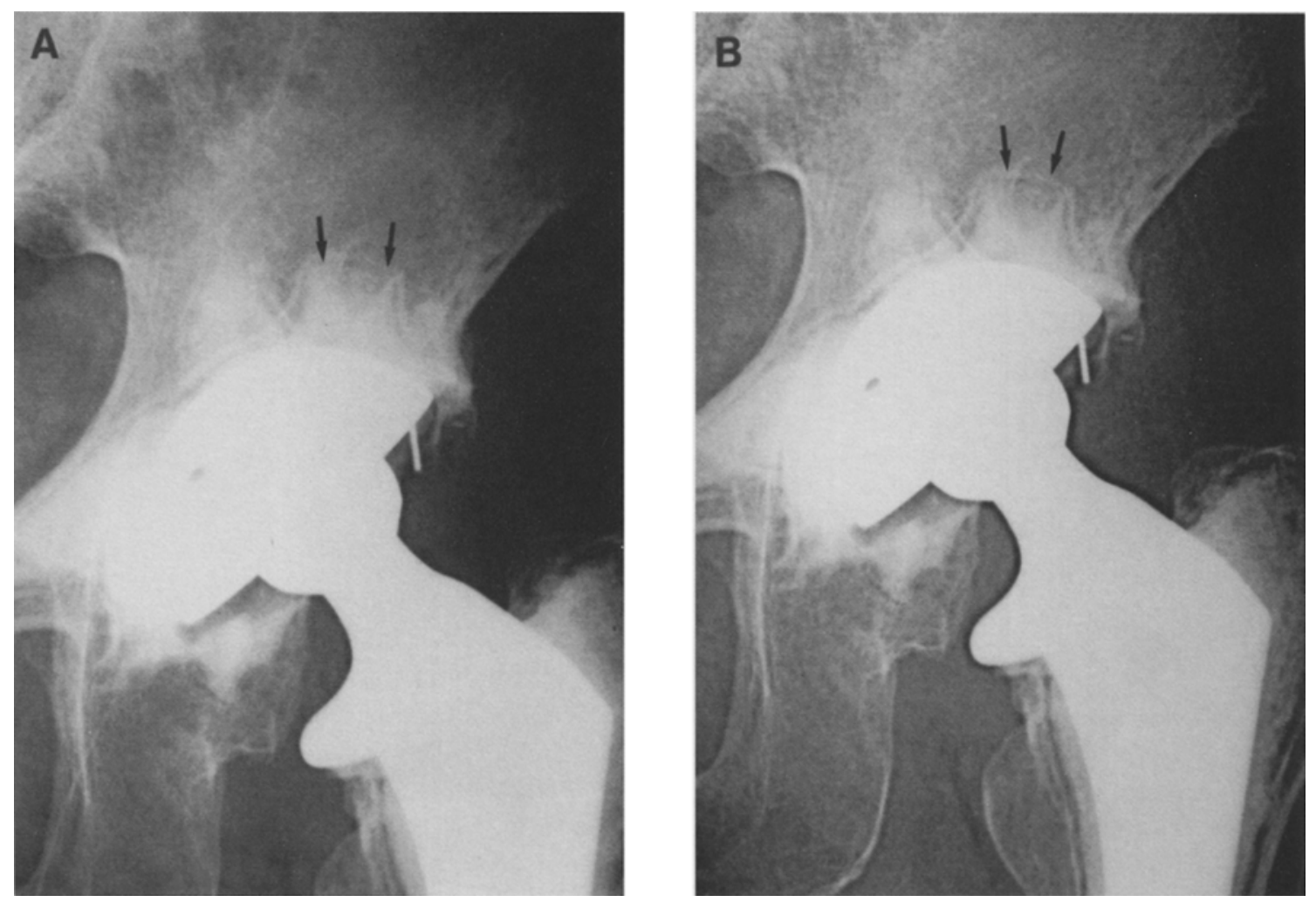

Fig 12. Case 9: The clear zone around the cup (arrow) on the default image (A) and on the edge-enhanced image (B). 

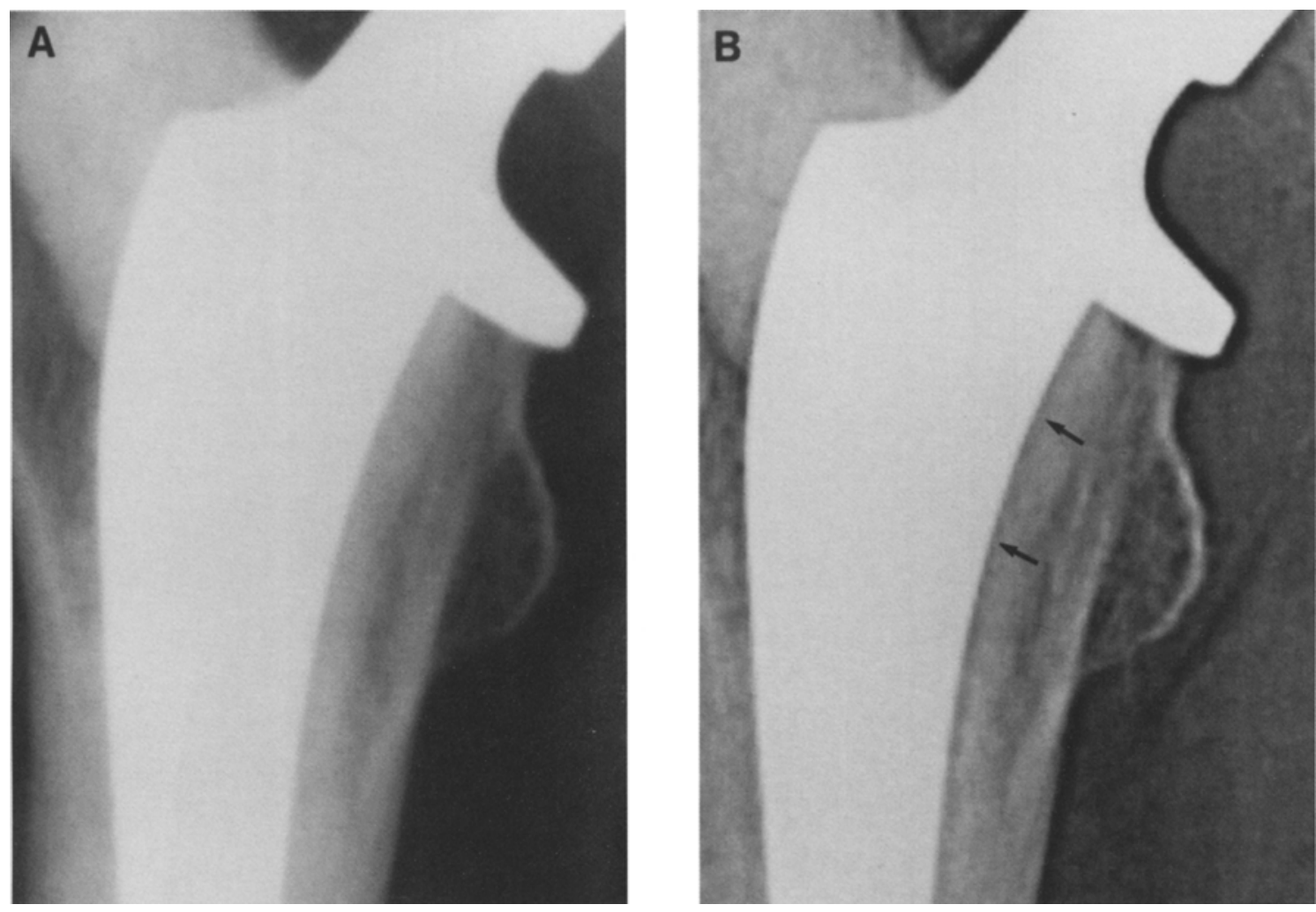

Fig 13. Case 10: There was no clear zone on the default image (A), but a radiolucent line had formed around the stem on the edge-enhanced image because of frequency processing (arrow) (B).
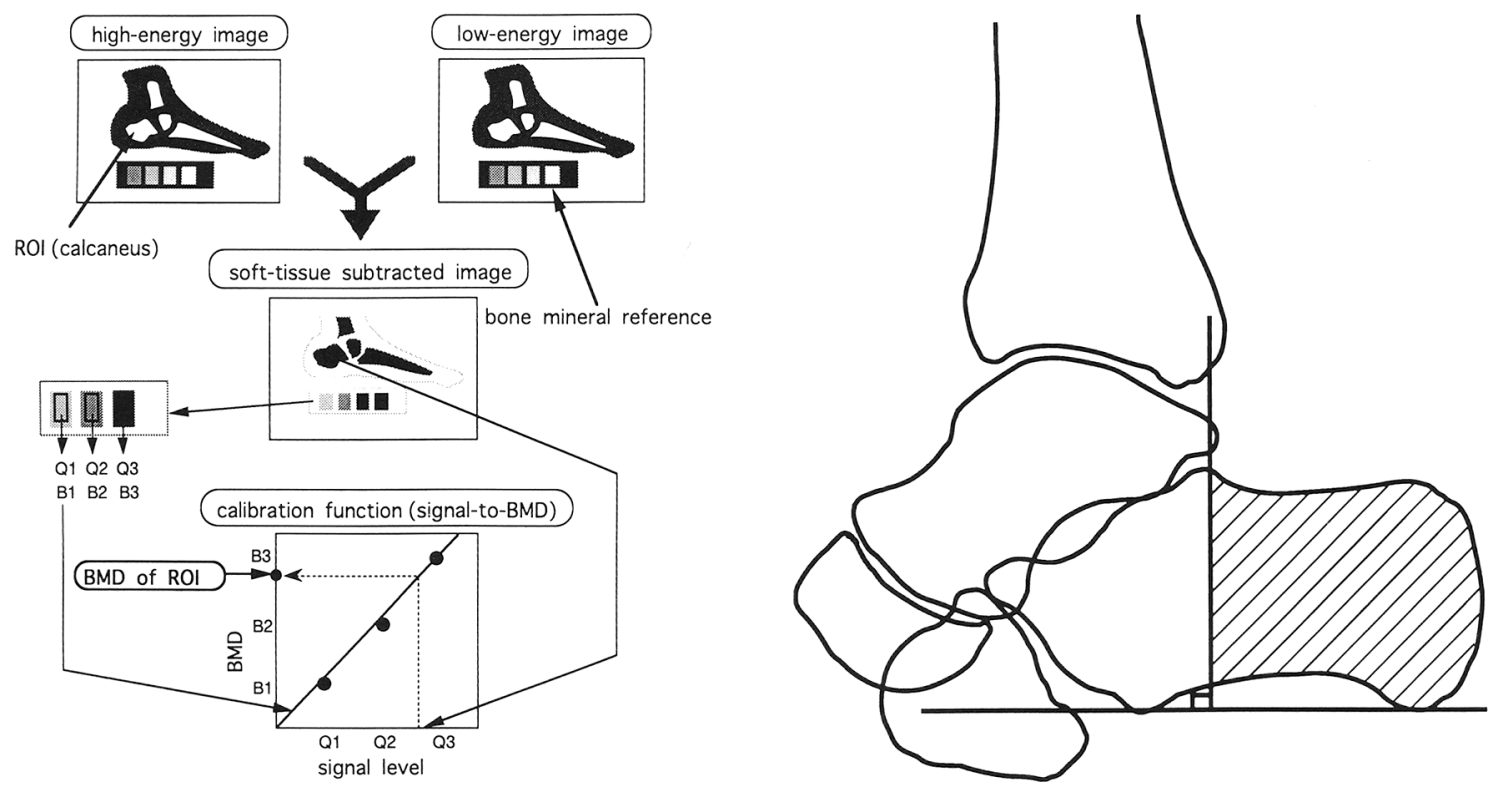

Fig 14. Description of CR-DXA

Fig 15. ROI in the calcaneus. 
Table 1. Repetitive Precision

\begin{tabular}{lcl}
\hline & DXA $\left(\mathrm{g} / \mathrm{cm}^{2}\right)$ & CR-DXA \\
\hline 1 & 0.609 & 0.561 \\
2 & 0.614 & 0.570 \\
3 & 0.614 & 0.571 \\
4 & 0.619 & 0.574 \\
5 & 0.614 & 0.574 \\
Average & 0.614 & 0.570 \\
SD & 0.0035 & 0.0053 \\
Precision (CV) & $0.57 \%$ & $0.94 \%$ \\
\hline
\end{tabular}

\section{ACKNOWLEDGMENT}

We thank Dr K. Satoh and the staff of the Department of Radiology, Osaka Prefectural Hospital for their valuable criticism and advise regarding this study.

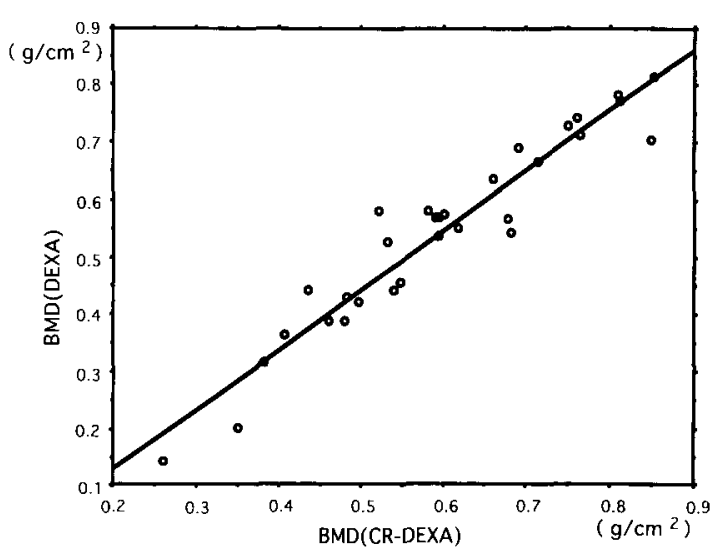

Fig 16. Correlation of the CR-DXA and DXA. $Y=1.048 X-$ $0.081 ; r=0.96 ; P<.0001$.

\section{REFERENCES}

1. Sonoda M, Takano M, Miyahara J: Computed radiography utilizing scanning laser stimulated luminescence. Radiology 148:833-838, 1983

2. Shimura K, Nakajima $\mathbf{N}$, Tanaka $\mathrm{H}$, et al: Basic investigation of dual-energy $\mathrm{x}$-ray absorptiometry for bone densitometry using computed radiography. Proc SPIE 1896 : 121-129, 1993 